\title{
Structure Effect of Imidazolium-Based Dicationic Ionic Liquids on Claisen Rearrangement
}

\section{Elina Priede,* Ilva Nakurte, and Andris Zicmanis}

Email: priede_elina@inbox.lv

University of Latvia, Faculty of Chemistry, 19, Rainis Blvd., Riga, 1586 Latvia

\section{SUPPLEMENTARY INFORMATION}

\section{Instruments}

The melting points of DILs were determined by Stuart SMP3 apparatus. UV-VIS spectra were recorded on UV/VIS Spectrometer Lambda 25. High-resolution mass spectra were obtained with Agilent 6230 TOF LC/MS instrument. Negative-ion ESI-MS analysis was performed on Agilent 6230 TOF LC/MS mass spectrometer. ESI-MS spectra were obtained for the solutions $\left(10^{-3} \mathrm{M}\right)$ of DILs in acetonitrile-water $(1: 1, \mathrm{v}: \mathrm{v}) .5 \mu \mathrm{L}$ of the samples were injected directly into the spectrometer at a constant flow $5 \mu \mathrm{L} / \mathrm{min}$. Source was used with the following parameters: negative ionization mode, drying gas flow $10 \mathrm{~mL} / \mathrm{min}$ and $200{ }^{\circ} \mathrm{C}$ temperature, fragmentor ionization was set in a range of 0 to $130 \mathrm{~V} . \mathrm{N}_{2}$ was used as the sheath gas at flow $8 \mathrm{ml} / \mathrm{min}$ and $200{ }^{\circ} \mathrm{C}$ temperature. The mass scan range was from $\mathrm{m} / \mathrm{z} 50$ to 3000 . Peak detection and spectrum extraction were performed with MassHunter Software (Agilent). Thermogravimetric analysis was performed by SII Extar6000 TG-DTA6300 under nitrogen atmosphere (temperature gradient $50 \ldots 500{ }^{\circ} \mathrm{C}$, heating rate $\left.-10{ }^{\circ} \mathrm{C} / \mathrm{min}\right)$. The products of the Claisen rearrangement were identified by GC (Shimadzu GC-2010) using Restek Rtx®)-1 Ms column (30 m x 0,25 mm x 0,25 $\mu \mathrm{m}$ ) equipped with mass spectrometer (Shimadzu GC/MS-QP2010). Quantitative analysis of the reaction mixture was performed by GC (YL6100 GC) using the same column equipped with a flame ionization detector (FID). Helium was exploited as the carrier gas (flow rate $-1 \mathrm{~mL} / \mathrm{min}$ ). 
Pure 2-allylphenol (4) was isolated by flash chromatography using Isolera One (Biotage) instrument, equipped with a silica gel column SNAP $10 \mathrm{~g}$ (Biotage).

\section{Characterization Data of Dicationic Ionic Liquids}

\section{Scheme S1}

\section{Scheme S2}

1,3-Bis(3-butylimidazolium-1-yl)propane dibromide (5a). Prepared from 1-butylimidazole (6a) and 1,3-dibromopropane (7a), obtained as a white solid, 85\% yield. M.p. $124-125{ }^{\circ} \mathrm{C} .{ }^{1} \mathrm{H}$ NMR (DMSO-d $\left.d_{6}\right) \delta .91\left(6 \mathrm{H}, \mathrm{t}, J=7.4 \mathrm{~Hz}, \underline{\mathrm{CH}}_{3} \mathrm{CH}_{2}\right), 1.28\left(4 \mathrm{H}\right.$, sext, $\left.J=7.4 \mathrm{~Hz}, \mathrm{CH}_{3} \underline{\mathrm{CH}}_{2} \mathrm{CH}_{2}\right)$, 1.79 (4H, quint, $J=7.4 \mathrm{~Hz}, \mathrm{C}_{2} \mathrm{H}_{5} \mathrm{C}_{2} \mathrm{CH}_{2}$ ), 2.43 (2H, quint, $J=7.1 \mathrm{~Hz}, \mathrm{NCH}_{2} \mathrm{CH}_{2} \mathrm{CH}_{2} \mathrm{~N}$ ), 4.22 $\left(8 \mathrm{H}, \mathrm{dt}, J=30.3,7.1 \mathrm{~Hz}, \mathrm{C}_{3} \mathrm{H}_{7} \underline{\mathrm{CH}}_{2} \mathrm{~N}, \mathrm{NC}_{2} \mathrm{CH}_{2} \underline{\mathrm{C}}_{2} \mathrm{~N}\right), 7.85(4 \mathrm{H}, \mathrm{d}, J=1.6 \mathrm{~Hz}, \mathrm{NC} \underline{\mathrm{HC}} \underline{\mathrm{HN}})$, 9.34 (2H, s, NCHN). HRMS (ESI): $\mathrm{MH}^{+}$, found 289.2401. $\left[\mathrm{C}_{17} \mathrm{H}_{29} \mathrm{~N}_{4}\right]^{+}$requires 289.2387.

1,4-Bis(3-butylimidazolium-1-yl)butane dichloride (5b). Prepared from 1-butylimidazole (6a) and 1,4-dichlorobutane (7b). The reaction mixture was heated in toluene $(25 \mathrm{~mL})$ at $95{ }^{\circ} \mathrm{C}$ for 24 $\mathrm{h}$ and the product was washed with boiling toluene $(3 \times 15 \mathrm{~mL})$. Obtained as a viscous, slightly yellow substance, $72 \%$ yield. ${ }^{1} \mathrm{H}$ NMR $\left(\mathrm{DMSO}_{-} d_{6}\right) \delta 0.89\left(6 \mathrm{H}, \mathrm{t}, J=7.4 \mathrm{~Hz}, \mathrm{C}_{3} \mathrm{CH}_{2}\right), 1.25(4 \mathrm{H}$, sext, $\left.J=7.5 \mathrm{~Hz}, \mathrm{CH}_{3} \underline{\mathrm{CH}}_{2} \mathrm{CH}_{2}\right), 1.87-1.72\left(8 \mathrm{H}, \mathrm{m}, \mathrm{C}_{2} \mathrm{H}_{5} \mathrm{C}_{2} \mathrm{CH}_{2}, \mathrm{NCH}_{2} \underline{\mathrm{CH}}_{2} \mathrm{C}_{2} \mathrm{CH}_{2} \mathrm{~N}\right.$, ), 4.20 $\left(4 \mathrm{H}, \mathrm{t}, J=7.2 \mathrm{~Hz}, \mathrm{C}_{3} \mathrm{H}_{7} \underline{\mathrm{C}}_{2} \mathrm{~N}\right), 4.29\left(4 \mathrm{H}, \mathrm{t}, J=6.0 \mathrm{~Hz}, \mathrm{NC}_{2}\left(\mathrm{CH}_{2}\right)_{2} \underline{\mathrm{C}}_{2} \mathrm{~N}\right), 7.89-7.83(2 \mathrm{H}, \mathrm{m}$, NCHCHN), 7.95-7.89 (2H, m, NCHCㅌN), 9.70 (2H, s, NCㅌN). HRMS (ESI): $\mathrm{MH}^{+}$, found 303.2539. $\left[\mathrm{C}_{18} \mathrm{H}_{31} \mathrm{~N}_{4}\right]^{+}$requires 303.2543 .

1,6-Bis(3-butylimidazolium-1-yl)hexane dichloride (5c). Prepared from 1-butylimidazole (6a) and 1,6-dichlorohexane (7c). The reaction mixture was heated under reflux in 2-propanol (25 $\mathrm{mL})$ for $16 \mathrm{~h}$ and the product was washed with diethyl ether $(3 \times 15 \mathrm{~mL})$. Obtained as a viscous, slightly yellow substance, $87 \%$ yield. ${ }^{1} \mathrm{H}$ NMR (DMSO- $\left.d_{6}\right) \delta 0.90\left(6 \mathrm{H}, \mathrm{t}, J=7.4 \mathrm{~Hz}, \mathrm{CH}_{3} \mathrm{CH}_{2}\right)$, 
1.32-1.18 (8H, m, N( $\left.\left(\mathrm{CH}_{2}\right)_{2} \mathrm{C}_{2} \underline{\mathrm{C}}_{2}\left(\mathrm{CH}_{2}\right)_{2} \mathrm{~N}, \quad \mathrm{CH}_{3} \underline{\mathrm{CH}}_{2} \mathrm{CH}_{2}\right), \quad 1.87-1.71 \quad(8 \mathrm{H}, \quad \mathrm{m}$, $\left.\mathrm{NCH}_{2} \underline{\mathrm{C}}_{2}\left(\mathrm{CH}_{2}\right)_{2} \mathrm{C}_{2} \mathrm{CH}_{2} \mathrm{~N}, \quad \mathrm{C}_{2} \mathrm{H}_{5} \mathrm{C}_{2} \mathrm{CH}_{2}\right), 4.18\left(8 \mathrm{H}, \quad\right.$ td, $J=7.2,2.4 \mathrm{~Hz}, \mathrm{C}_{3} \mathrm{H}_{7} \underline{\mathrm{CH}}_{2} \mathrm{~N}_{\text {, }}$ $\left.\mathrm{NC}_{2}\left(\mathrm{CH}_{2}\right)_{4} \underline{\mathrm{C}}_{2} \mathrm{~N}\right), 7.83-7.80(2 \mathrm{H}, \mathrm{m}, \mathrm{NC} \underline{\mathrm{HCHN}}), 7.86-7.83$ (2H, m, NCHCㅍN), 9.42 (2H, s, $\mathrm{NCHN}$ ). HRMS (ESI): $\mathrm{MH}^{+}$, found 331.2854. $\left[\mathrm{C}_{20} \mathrm{H}_{35} \mathrm{~N}_{4}\right]^{+}$requires 331.2856.

1,3-Bis(3-methylimidazolium-1-yl)propane dibromide (5d). Prepared from 1-methylimidazole (6b) and 1,3-dibromopropane (7a), obtained as a white solid, 92\% yield. M.p. 161-162 ${ }^{\circ} \mathrm{C}$ (lit. 161-162 $\left.{ }^{\circ} \mathrm{C}\right) .{ }^{[1] ~}{ }^{1} \mathrm{H}$ NMR (DMSO-d 6 ) $\delta 2.39\left(2 \mathrm{H}\right.$, quint, $\left.J=6.8 \mathrm{~Hz}, \mathrm{NCH}_{2} \mathrm{CH}_{2} \mathrm{CH}_{2} \mathrm{~N}\right), 3.87(6 \mathrm{H}, \mathrm{s}$,

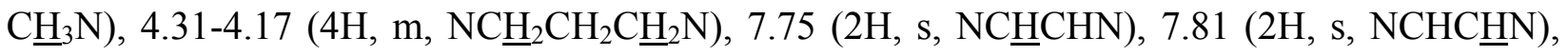
$9.24(2 \mathrm{H}, \mathrm{s}, \mathrm{NC} \underline{\mathrm{HN}}) . \mathrm{HRMS}(\mathrm{ESI}): \mathrm{MH}^{+}$, found 205.1438. $\left[\mathrm{C}_{11} \mathrm{H}_{17} \mathrm{~N}_{4}\right]^{+}$requires 205.1448 .

1,4-Bis(3-methylimidazolium-1-yl)butane dichloride (5e). Prepared from 1-methylimidazole (6b) and 1,4-dichlorobutane (7b), obtained as a white solid, 92\% yield. M.p. $182-183{ }^{\circ} \mathrm{C} .{ }^{1} \mathrm{H}$ NMR (DMSO-d $\left.d_{6}\right) \delta$ 1.86-1.73 (4H, m, $\left.\mathrm{NCH}_{2} \underline{\mathrm{C}}_{2} \underline{\mathrm{C}}_{2} \mathrm{CH}_{2} \mathrm{~N}\right), 3.88\left(6 \mathrm{H}, \mathrm{s}, \mathrm{C}_{3} \mathrm{~N}\right), 4.28(4 \mathrm{H}, \mathrm{s}$,

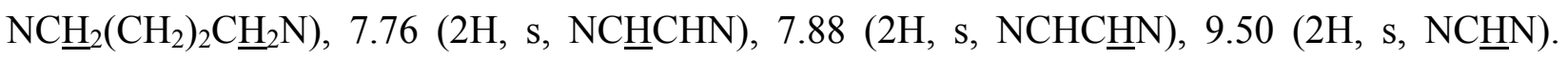
HRMS (ESI): $\mathrm{MH}^{+}$, found 219.1594. $\left[\mathrm{C}_{12} \mathrm{H}_{19} \mathrm{~N}_{4}\right]^{+}$requires 219.1604.

1,6-Bis(3-methylimidazolium-1-yl)hexane dichloride (5f). Prepared from 1-methylimidazole (6b) and 1,6-dichlorohexane (7c), obtained as a white solid, 87\% yield. M.p. 154-156 ${ }^{\circ} \mathrm{C}$ (lit. 154-156 $\left.{ }^{\circ} \mathrm{C}\right) .{ }^{[1]}{ }^{1} \mathrm{H}$ NMR (DMSO-d $) \delta$ 1.33-1.19 (4H, m, N $\left.\left(\mathrm{CH}_{2}\right)_{2} \mathrm{C}_{2} \mathrm{C}_{2} \underline{\mathrm{H}}_{2}\left(\mathrm{CH}_{2}\right)_{2} \mathrm{~N}\right), 1.85-1.71$ $\left(4 \mathrm{H}, \quad \mathrm{m}, \quad \mathrm{NCH}_{2} \underline{\mathrm{C}}_{2}\left(\mathrm{CH}_{2}\right)_{2} \mathrm{C}_{2} \mathrm{CH}_{2} \mathrm{~N}\right), 3.87\left(6 \mathrm{H}, \mathrm{s}, \mathrm{C}_{3} \mathrm{~N}\right), 4.19(4 \mathrm{H}, \mathrm{t}, J=7.1 \mathrm{~Hz}$,

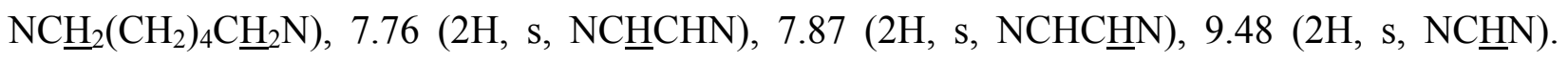
HRMS (ESI): $\mathrm{MH}^{+}$, found 247.1929. $\left[\mathrm{C}_{14} \mathrm{H}_{23} \mathrm{~N}_{4}\right]^{+}$requires 247.1917.

1,3-Bis(3-butyl-2-methylimidazolium-1-yl)propane dibromide (9a). Prepared from 1-butyl-2methylimidazole (8a) and 1,3-dibromopropane (7a). The reaction mixture was heated under reflux in 2-propanol $(25 \mathrm{~mL})$ for $24 \mathrm{~h}$ and the product was washed with boiling toluene $(3 \mathrm{x} 15$ $\mathrm{mL})$. Obtained as a viscous, slightly brown substance, $64 \%$ yield. ${ }^{1} \mathrm{H}$ NMR $\left(\mathrm{DMSO}-d_{6}\right) \delta 0.95-$ 
$0.86\left(6 \mathrm{H}, \mathrm{m}, \mathrm{CH}_{3} \mathrm{CH}_{2}\right), 1.30\left(4 \mathrm{H}\right.$, sext, $\left.J=7.5 \mathrm{~Hz}, \mathrm{CH}_{3} \mathrm{CH}_{2} \mathrm{CH}_{2}\right), 1.77-1.63$ (4H, m, $\left.\mathrm{C}_{2} \mathrm{H}_{5} \mathrm{C}_{2} \mathrm{CH}_{2}\right), 2.34-2.20\left(2 \mathrm{H}, \mathrm{m}, \mathrm{NCH}_{2} \underline{\mathrm{CH}}_{2} \mathrm{CH}_{2} \mathrm{~N}\right), 2.67\left(6 \mathrm{H}, \mathrm{s}, \mathrm{C}_{3} \mathrm{C}\right), 4.12(4 \mathrm{H}, \mathrm{t}, J=7.4 \mathrm{~Hz}$, $\left.\mathrm{C}_{3} \mathrm{H}_{7} \underline{\mathrm{C}}_{2} \mathrm{~N}\right), 4.24\left(4 \mathrm{H}, \mathrm{t}, J=7.5 \mathrm{~Hz}, \mathrm{NC}_{2} \mathrm{CH}_{2} \underline{\mathrm{C}}_{2} \mathrm{~N}\right), 7.75(4 \mathrm{H}, \mathrm{dd}, J=14.5,2.1 \mathrm{~Hz}$,

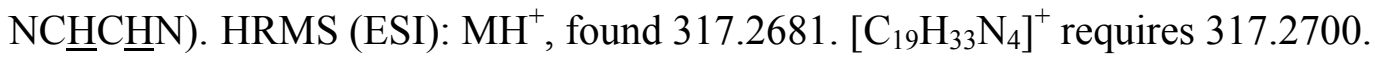

1,3-Bis(2,3-dimethylimidazolium-1-yl)propane dibromide (9b). Prepared from 1,2dimethylimidazole (8b) and 1,3-dibromopropane (7a). The reaction mixture was heated under reflux in 2-propanol $(25 \mathrm{~mL})$ for $16 \mathrm{~h}$ and the product was washed with cold 2-propanol $(2 \times 10$ mL). Obtained as a white solid, 92\% yield. M.p. $293-294{ }^{\circ} \mathrm{C}$ (decomp.) (lit. $\left.298{ }^{\circ} \mathrm{C}\right) .{ }^{[1]}{ }^{1} \mathrm{H}$ NMR $\left(\mathrm{DMSO}-d_{6}\right) \delta 2.21\left(2 \mathrm{H}\right.$, quint, $\left.J=7.5 \mathrm{~Hz}, \mathrm{NCH}_{2} \underline{\mathrm{C}}_{2} \mathrm{CH}_{2} \mathrm{~N}\right), 2.62\left(6 \mathrm{H}, \mathrm{s}, \mathrm{C}_{3} \mathrm{C}\right), 3.76(6 \mathrm{H}, \mathrm{s}$, $\left.\mathrm{C}_{3} \mathrm{~N}\right), 4.22\left(4 \mathrm{H}, \mathrm{t}, J=7.4 \mathrm{~Hz}, \mathrm{NCH}_{2} \mathrm{CH}_{2} \underline{\mathrm{CH}}_{2} \mathrm{~N}\right), 7.66(2 \mathrm{H}, \mathrm{d}, J=2.1 \mathrm{~Hz}, \mathrm{NC} \underline{\mathrm{HCHN}}), 7.71-7.67$ $(2 \mathrm{H}, \mathrm{m}, \mathrm{NCHC} \underline{\mathrm{HN}}) . \mathrm{HRMS}(\mathrm{ESI}): \mathrm{MH}^{+}$, found 233.1755. $\left[\mathrm{C}_{13} \mathrm{H}_{21} \mathrm{~N}_{4}\right]^{+}$requires 233.1761 .

1,4-Bis(2,3-dimethylimidazolium-1-yl)butane dichloride $\quad(9 c) . \quad$ Prepared from 1,2dimethylimidazole (8b) and 1,4-dichlorobutane (7b) in a similar way as $\mathbf{9 b}$, obtained as a white solid, $\quad 60 \%$ yield. $\quad$ M.p. $\quad 249-250 \quad{ }^{\circ} \mathrm{C} . \quad{ }^{1} \mathrm{H} \quad \mathrm{NMR} \quad\left(\mathrm{DMSO}-\mathrm{d}_{6}\right) \quad \delta \quad 1.79-1.66 \quad(4 \mathrm{H}, \quad \mathrm{m}$, $\left.\mathrm{NCH}_{2} \underline{\mathrm{C}}_{2} \underline{\mathrm{C}}_{2} \mathrm{CH}_{2} \mathrm{~N}\right), 2.61\left(6 \mathrm{H}, \mathrm{s}, \mathrm{CH}_{3} \mathrm{C}\right), 3.76\left(6 \mathrm{H}, \mathrm{s}, \mathrm{C}_{3} \mathrm{~N}\right), 4.19(4 \mathrm{H}, \mathrm{t}, J=5.8 \mathrm{~Hz}$, $\left.\mathrm{NC}_{2}\left(\mathrm{CH}_{2}\right)_{2} \underline{\mathrm{CH}}_{2} \mathrm{~N}\right), 7.69(4 \mathrm{H}, \mathrm{dd}, J=24.0,2.0 \mathrm{~Hz}, \mathrm{NC} \underline{\mathrm{HC}} \underline{\mathrm{HN}}) . \mathrm{HRMS}(\mathrm{ESI}): \mathrm{MH}^{+}$, found 247.1896. $\left[\mathrm{C}_{14} \mathrm{H}_{23} \mathrm{~N}_{4}\right]^{+}$requires 247.1917.

1,6-Bis(2,3-dimethylimidazolium-1-yl)hexane dichloride (9d). Prepared from 1,2dimethylimidazole (8b) and 1,6-dichlorohexane (7c) in a similar way as $\mathbf{9 b}$, obtained as a white solid, 81\% yield. M.p. $149-150{ }^{\circ} \mathrm{C} .{ }^{1} \mathrm{H}$ NMR (DMSO-d 6 ) $\delta 1.29$ (4H, quint, $J=3.7 \mathrm{~Hz}$, $\left.\mathrm{N}\left(\mathrm{CH}_{2}\right)_{2} \mathrm{CH}_{2} \mathrm{C}_{2}\left(\mathrm{CH}_{2}\right)_{2} \mathrm{~N}\right), 1.70$ (4H, quint, $\left.J=7.0 \mathrm{~Hz}, \mathrm{NCH}_{2} \underline{\mathrm{C}}_{2}\left(\mathrm{CH}_{2}\right)_{2} \mathrm{CH}_{2} \mathrm{CH}_{2} \mathrm{~N}\right), 2.59(6 \mathrm{H}, \mathrm{s}$, $\left.\mathrm{C}_{3} \mathrm{C}\right), 3.76\left(6 \mathrm{H}, \mathrm{s}, \underline{\mathrm{C}}_{3} \mathrm{~N}\right), 4.11\left(4 \mathrm{H}, \mathrm{t}, J=7.3 \mathrm{~Hz}, \mathrm{NC}_{2}\left(\mathrm{CH}_{2}\right)_{4} \underline{\mathrm{CH}}_{2} \mathrm{~N}\right), 7.68(4 \mathrm{H}, \mathrm{dd}, J=17.4$,

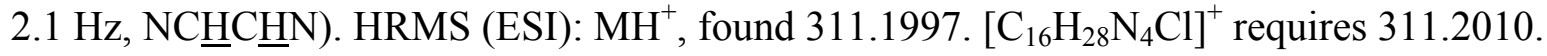


1,3-Bis(3-butylimidazolium-1-yl)propane bis(trifluoromethylsulfonyl)imide (1a). Prepared from 1,3-bis(3-butylimidazolium-1-yl)propane dibromide (5a) and lithium bis(trifluoromethylsulfonyl)imide, obtained as a viscous, slightly yellow liquid, 97\% yield. ${ }^{1} \mathrm{H}$ NMR (DMSO-d $) \delta 0.92\left(6 \mathrm{H}, \mathrm{t}, J=7.4 \mathrm{~Hz}, \underline{\mathrm{CH}}_{3} \mathrm{CH}_{2}\right), 1.28\left(4 \mathrm{H}\right.$, sext, $\left.J=7.5 \mathrm{~Hz}, \mathrm{CH}_{3} \mathrm{C}_{2} \mathrm{CH}_{2}\right)$, 1.79 (4H, quint, $J=7.4 \mathrm{~Hz}, \mathrm{C}_{2} \mathrm{H}_{5} \mathrm{C}_{2} \mathrm{CH}_{2}$ ), 2.40 (2H, quint, $J=7.1 \mathrm{~Hz}, \mathrm{NCH}_{2} \underline{\mathrm{CH}}_{2} \mathrm{CH}_{2} \mathrm{~N}$ ), 4.19 $\left(8 \mathrm{H}, \mathrm{dt}, J=21.5,7.1 \mathrm{~Hz}, \mathrm{C}_{3} \mathrm{H}_{7} \mathrm{CH}_{2} \mathrm{~N}, \mathrm{NCH}_{2} \mathrm{CH}_{2} \underline{\mathrm{C}}_{2} \mathrm{~N}\right), 7.77(2 \mathrm{H}, \mathrm{s}, \mathrm{NC} \underline{H C H N}), 7.82$ (2H, s, $\mathrm{NCHC} \underline{\mathrm{HN}}), 9.16(2 \mathrm{H}, \mathrm{s}, \mathrm{NC} \underline{\mathrm{HN}})$. HRMS (ESI): $\mathrm{MH}^{+}$, found 289.2372. $\left[\mathrm{C}_{17} \mathrm{H}_{29} \mathrm{~N}_{4}\right]^{+}$requires 289.2387.

1,3-Bis(3-butylimidazolium-1-yl)propane hexafluorophosphate (1b). Prepared from 1,3-bis(3butylimidazolium-1-yl)propane dibromide (5a) and potassium hexafluorophosphate, obtained as a white solid, 79\% yield. M.p. 96-97 ${ }^{\circ} \mathrm{C}$ (lit. 96-98 $\left.{ }^{\circ} \mathrm{C}\right) .{ }^{[3]}{ }^{1} \mathrm{H}$ NMR (DMSO- $\left.d_{6}\right) \delta 0.92(6 \mathrm{H}, \mathrm{t}, J=$ $\left.7.4 \mathrm{~Hz}, \underline{\mathrm{C}}_{3} \mathrm{CH}_{2}\right), 1.28\left(4 \mathrm{H}, \mathrm{sext}, J=7.6 \mathrm{~Hz}, \mathrm{CH}_{3} \underline{\mathrm{C}}_{2} \mathrm{CH}_{2}\right), 1.86-1.68\left(4 \mathrm{H}, \mathrm{m}, \mathrm{C}_{2} \mathrm{H}_{5} \mathrm{C}_{2} \mathrm{CH}_{2}\right)$, $2.39\left(2 \mathrm{H}\right.$, quint, $\left.J=7.1 \mathrm{~Hz}, \mathrm{NCH}_{2} \underline{\mathrm{CH}}_{2} \mathrm{CH}_{2} \mathrm{~N}\right), 4.19\left(8 \mathrm{H}, \mathrm{dt}, J=19.3,7.2 \mathrm{~Hz}, \mathrm{C}_{3} \mathrm{H}_{7} \mathrm{C}_{2} \mathrm{~N}_{\text {, }}\right.$

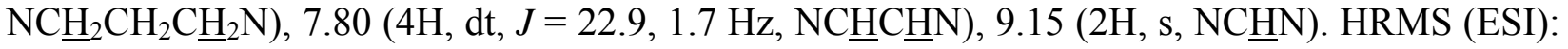
$\mathrm{MH}^{+}$, found 289.2368. $\left[\mathrm{C}_{17} \mathrm{H}_{29} \mathrm{~N}_{4}\right]^{+}$requires 289.2387.

1,4-Bis(3-butylimidazolium-1-yl)butane bis(trifluoromethylsulfonyl)imide (1c). Prepared from 1,4-bis(3-butylimidazolium-1-yl)butane dichloride (5b) and lithium bis(trifluoromethylsulfonyl)imide, obtained as a viscous, slightly yellow liquid, 94\% yield. ${ }^{1} \mathrm{H}$ NMR (DMSO-d 6 ) $\delta 0.91\left(6 \mathrm{H}, \mathrm{t}, J=7.4 \mathrm{~Hz}, \underline{\mathrm{C}}_{3} \mathrm{CH}_{2}\right), 1.27\left(4 \mathrm{H}\right.$, sext, $\left.J=7.5 \mathrm{~Hz}, \mathrm{CH}_{3} \underline{\mathrm{C}}_{2} \mathrm{CH}_{2}\right)$, 1.87-1.73 (8H, m, $\left.\mathrm{C}_{2} \mathrm{H}_{5} \mathrm{C}_{2} \mathrm{CH}_{2}, \quad \mathrm{NCH}_{2} \underline{\mathrm{C}}_{2} \mathrm{C}_{2} \mathrm{CH}_{2} \mathrm{~N}\right), \quad 4.26-4.11 \quad\left(8 \mathrm{H}, \quad \mathrm{m}, \mathrm{C}_{3} \mathrm{H}_{7} \mathrm{C}_{2} \mathrm{~N}\right.$,

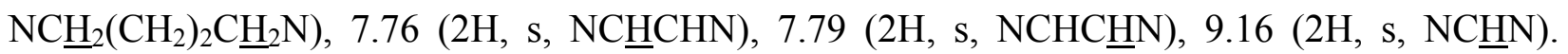
HRMS (ESI): $\mathrm{MH}^{+}$, found 303.2531. $\left[\mathrm{C}_{18} \mathrm{H}_{31} \mathrm{~N}_{4}\right]^{+}$requires 303.2543.

1,6-Bis(3-butylimidazolium-1-yl)hexane bis(trifluoromethylsulfonyl)imide (1d). Prepared from 1,6-bis(3-butylimidazolium-1-yl)hexane dichloride (5c) and lithium 
bis(trifluoromethylsulfonyl)imide, obtained as a viscous, slightly yellow liquid, $98 \%$ yield. ${ }^{1} \mathrm{H}$ NMR $\left(\right.$ DMSO- $\left.d_{6}\right) \quad \delta \quad 0.90 \quad\left(6 \mathrm{H}, \quad \mathrm{t}, \quad J=7.4 \quad \mathrm{~Hz}, \quad \underline{\mathrm{C}}_{3} \mathrm{CH}_{2}\right), \quad 1.36-1.17 \quad(8 \mathrm{H}, \quad \mathrm{m}$, $\left.\mathrm{N}\left(\mathrm{CH}_{2}\right)_{2} \underline{\mathrm{C}}_{2} \underline{\mathrm{C}}_{2}\left(\mathrm{CH}_{2}\right)_{2} \mathrm{~N}, \quad \mathrm{CH}_{3} \underline{\mathrm{C}}_{2} \mathrm{CH}_{2}\right), \quad 1.84-1.74 \quad\left(8 \mathrm{H}, \quad \mathrm{m}, \quad \mathrm{NCH}_{2} \underline{\mathrm{C}}_{2}\left(\mathrm{CH}_{2}\right)_{2} \underline{\mathrm{C}}_{2} \mathrm{CH}_{2} \mathrm{~N}\right.$, $\left.\mathrm{C}_{2} \mathrm{H}_{5} \mathrm{C}_{2} \mathrm{CH}_{2}\right), 4.23-4.09\left(8 \mathrm{H}, \mathrm{m}, \mathrm{C}_{3} \mathrm{H}_{7} \mathrm{C}_{2} \mathrm{~N}, \mathrm{NC}_{2}\left(\mathrm{CH}_{2}\right)_{4} \mathrm{C}_{2} \mathrm{~N}\right), 7.77(4 \mathrm{H}, \mathrm{dt}, J=6.9,1.8 \mathrm{~Hz}$, $\mathrm{NC} \underline{\mathrm{H}} \underline{\mathrm{HN}}), 9.16(2 \mathrm{H}, \mathrm{s}, \mathrm{NC} \underline{\mathrm{HN}})$. HRMS (ESI): $\mathrm{MH}^{+}$, found 331.2859. $\left[\mathrm{C}_{20} \mathrm{H}_{35} \mathrm{~N}_{4}\right]^{+}$requires 331.2856 .

1,3-Bis(3-methylimidazolium-1-yl)propane bis(trifluoromethylsulfonyl)imide (1e). Prepared from 1,3-bis(3-methylimidazolium-1-yl)propane dibromide (5d) and lithium bis(trifluoromethylsulfonyl)imide, obtained as a white solid, $85 \%$ yield. M.p. $49-50{ }^{\circ} \mathrm{C} .{ }^{1} \mathrm{H}$ NMR (DMSO-d $\left.)_{6}\right) \delta 2.36\left(2 \mathrm{H}\right.$, quint, $\left.J=7.0 \mathrm{~Hz}, \mathrm{NCH}_{2} \underline{\mathrm{CH}}_{2} \mathrm{CH}_{2} \mathrm{~N}\right), 3.86\left(6 \mathrm{H}, \mathrm{s}, \mathrm{C}_{3} \mathrm{~N}\right), 4.20(4 \mathrm{H}, \mathrm{t}, J=$

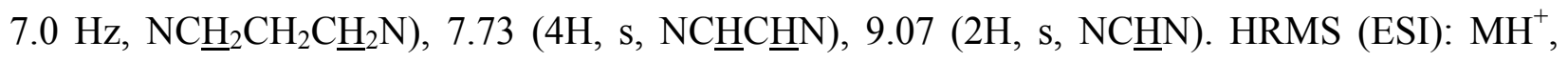
found 205.1458. $\left[\mathrm{C}_{11} \mathrm{H}_{17} \mathrm{~N}_{4}\right]^{+}$requires 205.1448.

1,3-Bis(3-methylimidazolium-1-yl)propane hexafluorophosphate (1f). Prepared from 1,3bis(3-methylimidazolium-1-yl)propane dibromide (5d) and potassium hexafluorophosphate, obtained as a white solid, $83 \%$ yield. M.p. $135-136{ }^{\circ} \mathrm{C}$ (lit. $131{ }^{\circ} \mathrm{C}$ ). ${ }^{[1]}{ }^{1} \mathrm{H}$ NMR (DMSO- $\left.d_{6}\right) \delta$ $2.36\left(2 \mathrm{H}\right.$, quint, $\left.J=7.0 \mathrm{~Hz}, \mathrm{NCH}_{2} \underline{\mathrm{C}}_{2} \mathrm{CH}_{2} \mathrm{~N}\right), 3.86\left(6 \mathrm{H}, \mathrm{s}, \underline{\mathrm{C}}_{3} \mathrm{~N}\right), 4.20(4 \mathrm{H}, \mathrm{t}, J=6.9 \mathrm{~Hz}$, $\left.\mathrm{NC}_{2} \mathrm{CH}_{2} \underline{\mathrm{C}}_{2} \mathrm{~N}\right), 7.74(4 \mathrm{H}, \mathrm{s}, \mathrm{NC} \underline{\mathrm{HC}} \underline{\mathrm{HN}}), 9.08(2 \mathrm{H}, \mathrm{s}, \mathrm{NC} \underline{\mathrm{HN}}) . \mathrm{HRMS}(\mathrm{ESI}): \mathrm{MH}^{+}$, found 205.1471. $\left[\mathrm{C}_{11} \mathrm{H}_{17} \mathrm{~N}_{4}\right]^{+}$requires 205.1448 .

1,4-Bis(3-methylimidazolium-1-yl)butane bis(trifluoromethylsulfonyl)imide (1g). Prepared from 1,4-bis(3-methylimidazolium-1-yl)butane dichloride (5e) and lithium bis(trifluoromethylsulfonyl)imide, obtained as a white solid, $93 \%$ yield. M.p. $57-58{ }^{\circ} \mathrm{C}$ (lit. $54-56$ $\left.{ }^{\circ} \mathrm{C}\right) .{ }^{[2] ~}{ }^{1} \mathrm{H}$ NMR (DMSO-d $\left.d_{6}\right) \delta$ 1.85-1.67 (4H, m, NCH$\left.{ }_{2} \underline{\mathrm{H}}_{2} \mathrm{C}_{2} \mathrm{CH}_{2} \mathrm{~N}\right), 3.85\left(6 \mathrm{H}, \mathrm{s}, \mathrm{C}_{3} \mathrm{~N}\right), 4.19$ $\left(4 \mathrm{H}, \mathrm{t}, J=6.0 \mathrm{~Hz}, \mathrm{NC}_{2}\left(\mathrm{CH}_{2}\right)_{2} \mathrm{C}_{2} \mathrm{~N}\right), 7.72(4 \mathrm{H}, \mathrm{dt}, J=9.0,1.8 \mathrm{~Hz}, \mathrm{NC} \underline{\mathrm{HC}} \underline{\mathrm{HN}}), 9.07(2 \mathrm{H}, \mathrm{s}$, $\mathrm{NC} \underline{\mathrm{HN}}$ ). HRMS (ESI): $\mathrm{MH}^{+}$, found 219.1588. $\left[\mathrm{C}_{12} \mathrm{H}_{19} \mathrm{~N}_{4}\right]^{+}$requires 219.1604 . 
1,6-Bis(3-methylimidazolium-1-yl)hexane bis(trifluoromethylsulfonyl)imide (1h). Prepared from 1,6-bis(3-methylimidazolium-1-yl)hexane dichloride (5f) and lithium bis(trifluoromethylsulfonyl)imide, obtained as a viscous, colorless liquid, 92\% yield. ${ }^{1} \mathrm{H}$ NMR $\left(\mathrm{DMSO}_{6}\right) \quad \delta \quad 1.35-1.18 \quad\left(4 \mathrm{H}, \quad \mathrm{m}, \quad \mathrm{N}\left(\mathrm{CH}_{2}\right)_{2} \mathrm{C}_{2} \underline{\mathrm{C}}_{2}\left(\mathrm{CH}_{2}\right)_{2} \mathrm{~N}\right), \quad 1.89-1.69 \quad(4 \mathrm{H}, \quad \mathrm{m}$, $\left.\mathrm{NCH}_{2} \underline{\mathrm{C}}_{2}\left(\mathrm{CH}_{2}\right)_{2} \underline{\mathrm{CH}}_{2} \mathrm{CH}_{2} \mathrm{~N}\right), 3.85\left(6 \mathrm{H}, \mathrm{s}, \underline{\mathrm{CH}}_{3} \mathrm{~N}\right), 4.14\left(4 \mathrm{H}, \mathrm{t}, J=7.2 \mathrm{~Hz}, \mathrm{NC}_{2}\left(\mathrm{CH}_{2}\right)_{4} \underline{\mathrm{CH}}_{2} \mathrm{~N}\right)$,

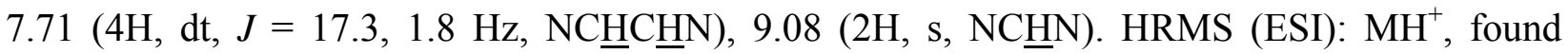
247.1901. $\left[\mathrm{C}_{14} \mathrm{H}_{23} \mathrm{~N}_{4}\right]^{+}$requires 247.1917 .

1,3-Bis(3-butyl-2-methylimidazolium-1-yl)propane bis(trifluoromethylsulfonyl)imide (4a). Prepared from 1,3-bis(3-butyl-2-methylimidazolium-1-yl)propane dibromide (9a) and lithium bis(trifluoromethylsulfonyl)imide, obtained as a viscous, slightly yellow liquid, $77 \%$ yield. ${ }^{1} \mathrm{H}$ NMR (DMSO-d 6 ) $\delta 0.92\left(6 \mathrm{H}, \mathrm{t}, J=7.4 \mathrm{~Hz}, \underline{\mathrm{CH}}_{3} \mathrm{CH}_{2}\right), 1.30\left(4 \mathrm{H}\right.$, sext, $\left.J=7.5 \mathrm{~Hz}, \mathrm{CH}_{3} \mathrm{C}_{2} \mathrm{CH}_{2}\right)$, 1.75-1.65 (4H, m, $\left.\mathrm{C}_{2} \mathrm{H}_{5} \mathrm{CH}_{2} \mathrm{CH}_{2}\right), 2.23\left(2 \mathrm{H}\right.$, quint, $\left.J=7.5 \mathrm{~Hz}, \mathrm{NCH}_{2} \underline{\mathrm{CH}}_{2} \mathrm{CH}_{2} \mathrm{~N}\right), 2.62(6 \mathrm{H}, \mathrm{s}$, $\left.\mathrm{C}_{3} \mathrm{C}\right), 4.11\left(4 \mathrm{H}, \mathrm{t}, J=7.4 \mathrm{~Hz}, \mathrm{C}_{3} \mathrm{H}_{7} \underline{\mathrm{C}}_{2} \mathrm{~N}\right), 4.17\left(4 \mathrm{H}, \mathrm{t}, J=7.6 \mathrm{~Hz}, \mathrm{NC}_{2} \mathrm{CH}_{2} \mathrm{C}_{2} \mathrm{~N}\right), 7.68(4 \mathrm{H}$, dd, $J=20.5,2.1 \mathrm{~Hz}, \mathrm{NC} \underline{\mathrm{HC}} \underline{\mathrm{HN}}$ ). HRMS (ESI): $\mathrm{MH}^{+}$, found 317.2682. $\left[\mathrm{C}_{19} \mathrm{H}_{33} \mathrm{~N}_{4}\right]^{+}$requires 317.2700.

\section{1,3-Bis(2,3-dimethylimidazolium-1-yl)propane bis(trifluoromethylsulfonyl)imide}

(4b).

Prepared from 1,3-bis(2,3-dimethylimidazolium-1-yl)propane dibromide (9b) and lithium bis(trifluoromethylsulfonyl)imide, obtained as a white solid, 93\% yield. M.p. $90-91{ }^{\circ} \mathrm{C}$ (lit. 91 $\left.{ }^{\circ} \mathrm{C}\right) .{ }^{[1]}{ }^{1} \mathrm{H}$ NMR $\left(\mathrm{DMSO}-d_{6}\right) \delta 2.20\left(2 \mathrm{H}\right.$, quint, $\left.J=7.5 \mathrm{~Hz}, \mathrm{NCH}_{2} \underline{\mathrm{H}}_{2} \mathrm{CH}_{2} \mathrm{~N}\right), 2.59\left(6 \mathrm{H}, \mathrm{s}, \mathrm{CH}_{3} \mathrm{C}\right)$, $3.75\left(6 \mathrm{H}, \mathrm{s}, \mathrm{C}_{3} \mathrm{~N}\right), 4.17\left(4 \mathrm{H}, \mathrm{t}, J=7.5 \mathrm{~Hz}, \mathrm{NC}_{2} \mathrm{CH}_{2} \underline{\mathrm{C}}_{2} \mathrm{~N}\right), 7.62(4 \mathrm{H}, \mathrm{dd}, J=12.7,2.1 \mathrm{~Hz}$, $\mathrm{NC} \underline{H C} \underline{H N}) . H R M S(E S I): \mathrm{MH}^{+}$, found 233.1762. $\left[\mathrm{C}_{13} \mathrm{H}_{21} \mathrm{~N}_{4}\right]^{+}$requires 233.1761 .

\section{1,4-Bis(2,3-dimethylimidazolium-1-yl)butane bis(trifluoromethylsulfonyl)imide $\quad$ (4c).}

Prepared from 1,4-bis(2,3-dimethylimidazolium-1-yl)butane dichloride (9c) and lithium bis(trifluoromethylsulfonyl)imide, obtained as a white solid, 94\% yield. M.p. $126-127{ }^{\circ} \mathrm{C} .{ }^{1} \mathrm{H}$ 
NMR (DMSO-d $\left.d_{6}\right) \delta$ 1.81-1.63 (4H, m, $\left.\mathrm{NCH}_{2} \underline{\mathrm{C}}_{2} \mathrm{C}_{2} \mathrm{CH}_{2} \mathrm{~N}\right), 2.57\left(6 \mathrm{H}, \mathrm{s}, \mathrm{C}_{3} \mathrm{C}\right), 3.75(6 \mathrm{H}, \mathrm{s}$, $\left.\underline{\mathrm{C}}_{3} \mathrm{~N}\right), 4.13\left(4 \mathrm{H}, \mathrm{s}, \mathrm{NC}_{2}\left(\mathrm{CH}_{2}\right)_{2} \underline{\mathrm{C}}_{2} \mathrm{~N}\right), 7.61(4 \mathrm{H}, \mathrm{dd}, J=7.5,2.1 \mathrm{~Hz}, \mathrm{NC} \underline{\mathrm{HC}} \underline{\mathrm{HN}}) . \mathrm{HRMS}$ (ESI): $\mathrm{MH}^{+}$, found 247.1924. $\left[\mathrm{C}_{14} \mathrm{H}_{23} \mathrm{~N}_{4}\right]^{+}$requires 247.1917.

\section{1,6-Bis(2,3-dimethylimidazolium-1-yl)hexane bis(trifluoromethylsulfonyl)imide}

(4d).

Prepared from 1,6-bis(2,3-dimethylimidazolium-1-yl)hexane dichloride (9d) and lithium bis(trifluoromethylsulfonyl)imide, obtained as a viscous, colorless liquid, $88 \%$ yield. ${ }^{1} \mathrm{H}$ NMR $\left(\mathrm{DMSO}_{6}\right) \delta 1.29\left(4 \mathrm{H}\right.$, quint, $\left.J=3.7 \mathrm{~Hz}, \mathrm{~N}\left(\mathrm{CH}_{2}\right)_{2} \mathrm{C}_{2} \underline{\mathrm{C}}_{2}\left(\mathrm{CH}_{2}\right)_{2} \mathrm{~N}\right), 1.76-1.62$ (4H, m, $\left.\mathrm{NCH}_{2} \underline{\mathrm{C}}_{2}\left(\mathrm{CH}_{2}\right)_{2} \mathrm{C}_{2} \mathrm{CH}_{2} \mathrm{~N}\right), 2.57\left(6 \mathrm{H}, \mathrm{s}, \underline{\mathrm{CH}}_{3} \mathrm{C}\right), 3.74\left(6 \mathrm{H}, \mathrm{s}, \underline{\mathrm{C}}_{3} \mathrm{~N}\right), 4.09(4 \mathrm{H}, \mathrm{t}, J=7.3 \mathrm{~Hz}$,

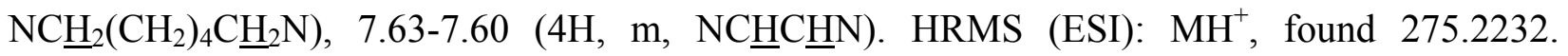
$\left[\mathrm{C}_{16} \mathrm{H}_{27} \mathrm{~N}_{4}\right]^{+}$requires 275.2230 .

\section{Table S1}

Figure S1

Figure S2

Figure S3

Figure S4

Figure S5

\section{REFERENCES}

[1] Anderson, J. L.; Ding, R.; Ellern, A.; Armstrong, D. W. J. Am. Chem. Soc. 2005, 127, 593604.

[2] Liu, Q.; Rantwijk, F.; Sheldon, R. A. J. Chem. Technol. Biotechnol. 2006, 81, 401-405.

[3] Lee, M.; Niu, Z.; Slebodnick, C.; Gibson, H. W. J. Phys. Chem. B 2010, 114, 7312-7319. 
Table S1. Thermal stability of the investigated imidazolium-based DILs

\begin{tabular}{|c|c|c|c|c|c|}
\hline Entry & DIL & Stability ${ }^{\mathrm{a}}\left({ }^{\circ} \mathrm{C}\right)$ & Entry & DIL & Stability $^{\mathrm{a}}\left({ }^{\circ} \mathrm{C}\right)$ \\
\hline 1 & $5 \mathbf{a}$ & 250 & 12 & $1 \mathrm{~g}$ & 410 \\
\hline 2 & 1b & 310 & 13 & $5 f$ & 250 \\
\hline 3 & $1 \mathrm{a}$ & 390 & 14 & $1 \mathrm{~h}$ & 400 \\
\hline 4 & $5 \mathbf{b}$ & 250 & 15 & $9 a$ & 260 \\
\hline 5 & $1 c$ & 400 & 16 & $\mathbf{4 a}$ & 380 \\
\hline 6 & $5 c$ & 210 & 17 & $9 \mathrm{~b}$ & 290 \\
\hline 7 & $1 d$ & 380 & 18 & $4 b$ & 410 \\
\hline 8 & $5 d$ & 290 & 19 & $9 c$ & 290 \\
\hline 9 & 1e & 400 & 20 & $4 c$ & 410 \\
\hline 10 & 1f & 330 & 21 & 9d & 270 \\
\hline 11 & $5 e$ & 300 & 22 & 4d & 400 \\
\hline
\end{tabular}

${ }^{\mathrm{a}}$ Temperature at which the decomposition starts. 
Scheme S1. Preparation of the investigated imidazolium-based DILs 1a-h and 5a-f.

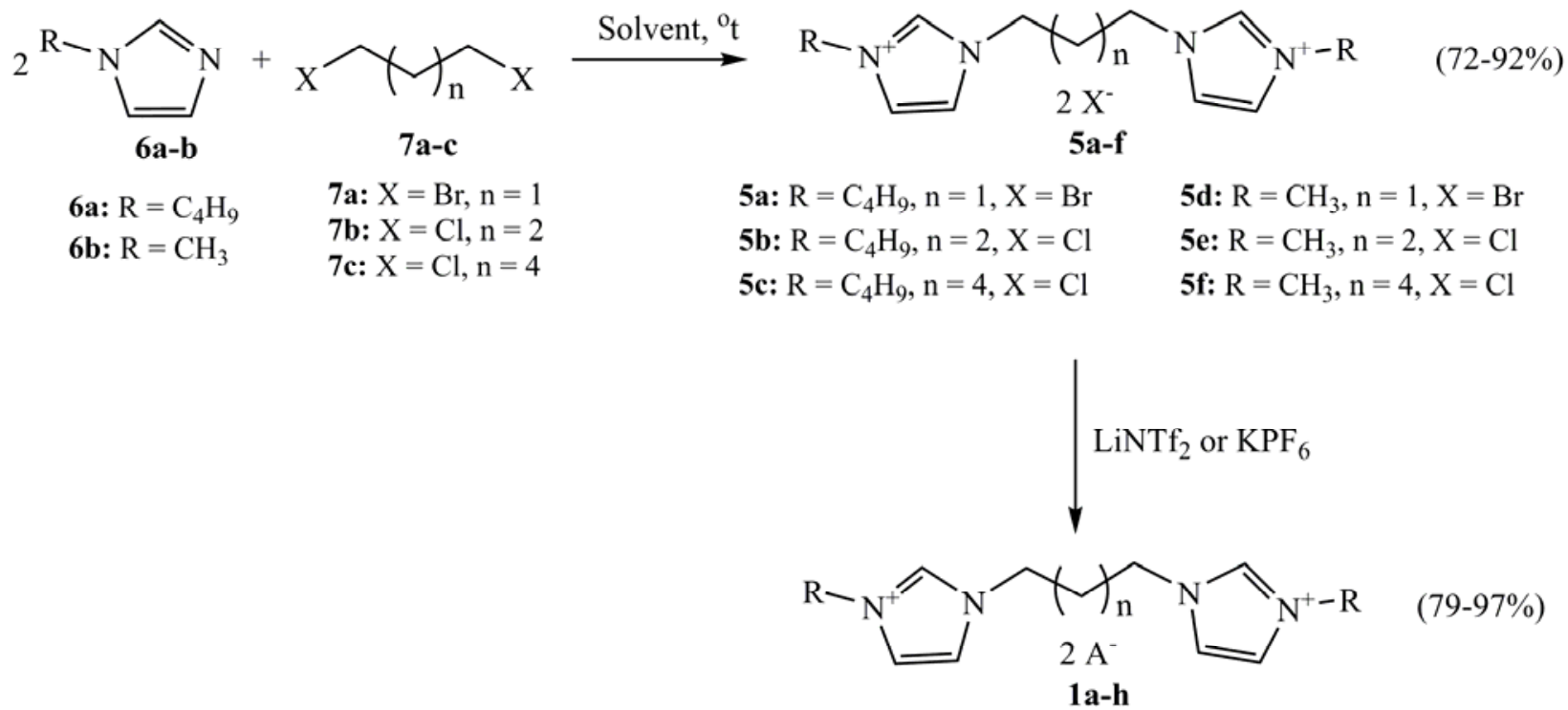
1a: $\mathrm{R}=\mathrm{C}_{4} \mathrm{H}_{9}, \mathrm{n}=1, \mathrm{~A}=\mathrm{NTf}_{2}$
1e: $\mathrm{R}=\mathrm{CH}_{3}, \mathrm{n}=1, \mathrm{~A}=\mathrm{NTf}_{2}$
1b: $\mathrm{R}=\mathrm{C}_{4} \mathrm{H}_{9}, \mathrm{n}=1, \mathrm{~A}=\mathrm{PF}_{6}$
1f: $\mathrm{R}=\mathrm{CH}_{3}, \mathrm{n}=1, \mathrm{~A}=\mathrm{PF}_{6}$
1c: $\mathrm{R}=\mathrm{C}_{4} \mathrm{H}_{9}, \mathrm{n}=2, \mathrm{~A}=\mathrm{NTf}_{2}$
1g: $\mathrm{R}=\mathrm{CH}_{3}, \mathrm{n}=2, \mathrm{~A}=\mathrm{NTf}_{2}$
1d: $\mathrm{R}=\mathrm{C}_{4} \mathrm{H}_{9}, \mathrm{n}=4, \mathrm{~A}=\mathrm{NTf}_{2}$
1h: $\mathrm{R}=\mathrm{CH}_{3}, \mathrm{n}=4, \mathrm{~A}=\mathrm{NTf}_{2}$ 
Scheme S2. Preparation of the investigated imidazolium-based DILs 4a-d and 9a-d.

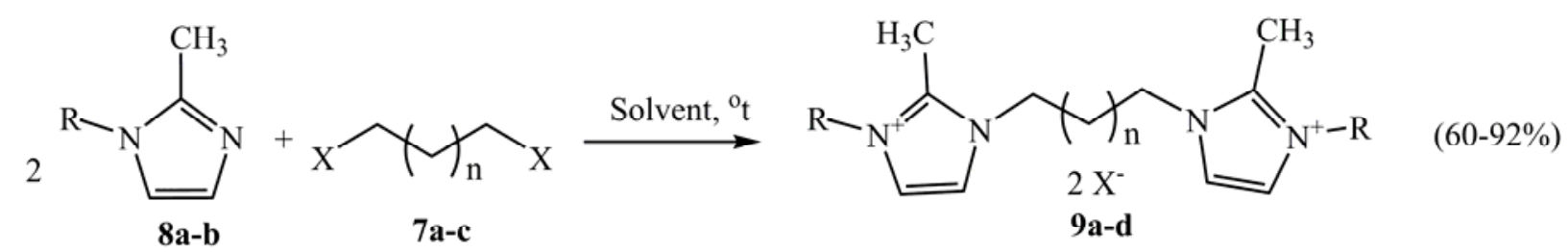

9a: $\mathrm{R}=\mathrm{C}_{4} \mathrm{H}_{9}, \mathrm{n}=1, \mathrm{X}=\mathrm{Br} \quad$ 9c: $\mathrm{R}=\mathrm{CH}_{3}, \mathrm{n}=2, \mathrm{X}=\mathrm{Cl}$

9b: $\mathrm{R}=\mathrm{CH}_{3}, \mathrm{n}=1, \mathrm{X}=\mathrm{Br} \quad$ 9d: $\mathrm{R}=\mathrm{CH}_{3}, \mathrm{n}=4, \mathrm{X}=\mathrm{Cl}$

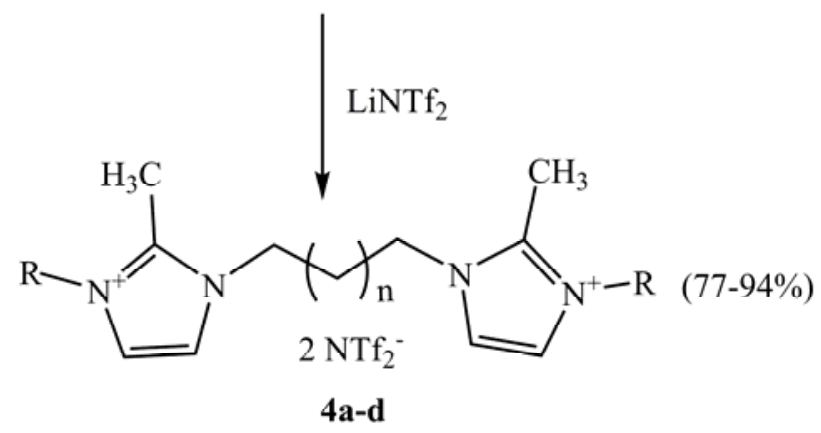

4a: $\mathrm{R}=\mathrm{C}_{4} \mathrm{H}_{9}, \mathrm{n}=1$

4b: $\mathrm{R}=\mathrm{CH}_{3}, \mathrm{n}=1$

4c: $\mathrm{R}=\mathrm{CH}_{3}, \mathrm{n}=2$

4d: $\mathrm{R}=\mathrm{CH}_{3}, \mathrm{n}=4$ 
Figure S1. Peak distribution in negative-ion ESI mass spectra (fragmentor voltage $-40 \mathrm{~V}$ ) of DILs 1e, 1f, 1h, 5a, and 5d.

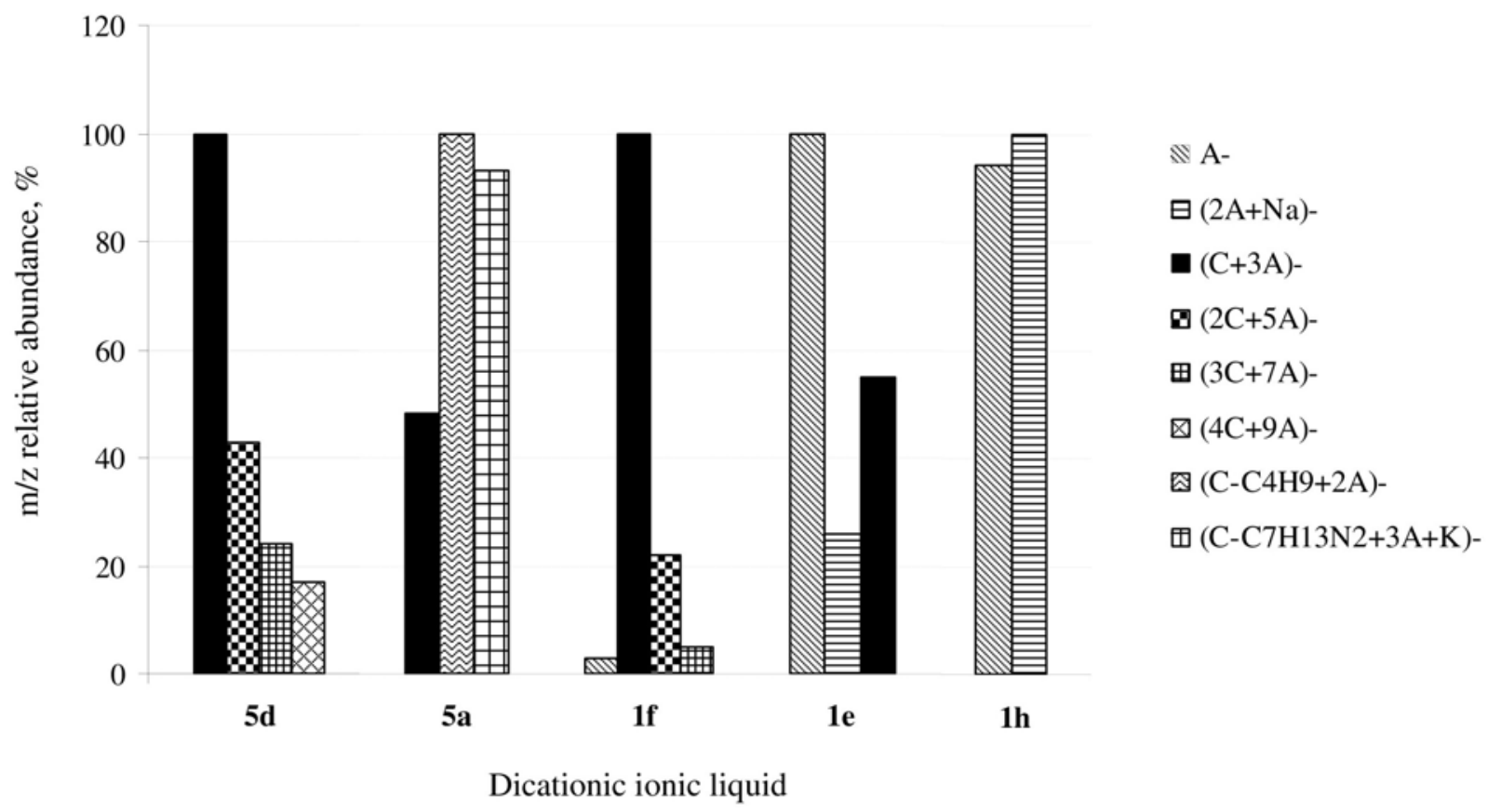


Figure S2. ${ }^{1} \mathrm{H}$ NMR spectrum of pure DIL 5 a.

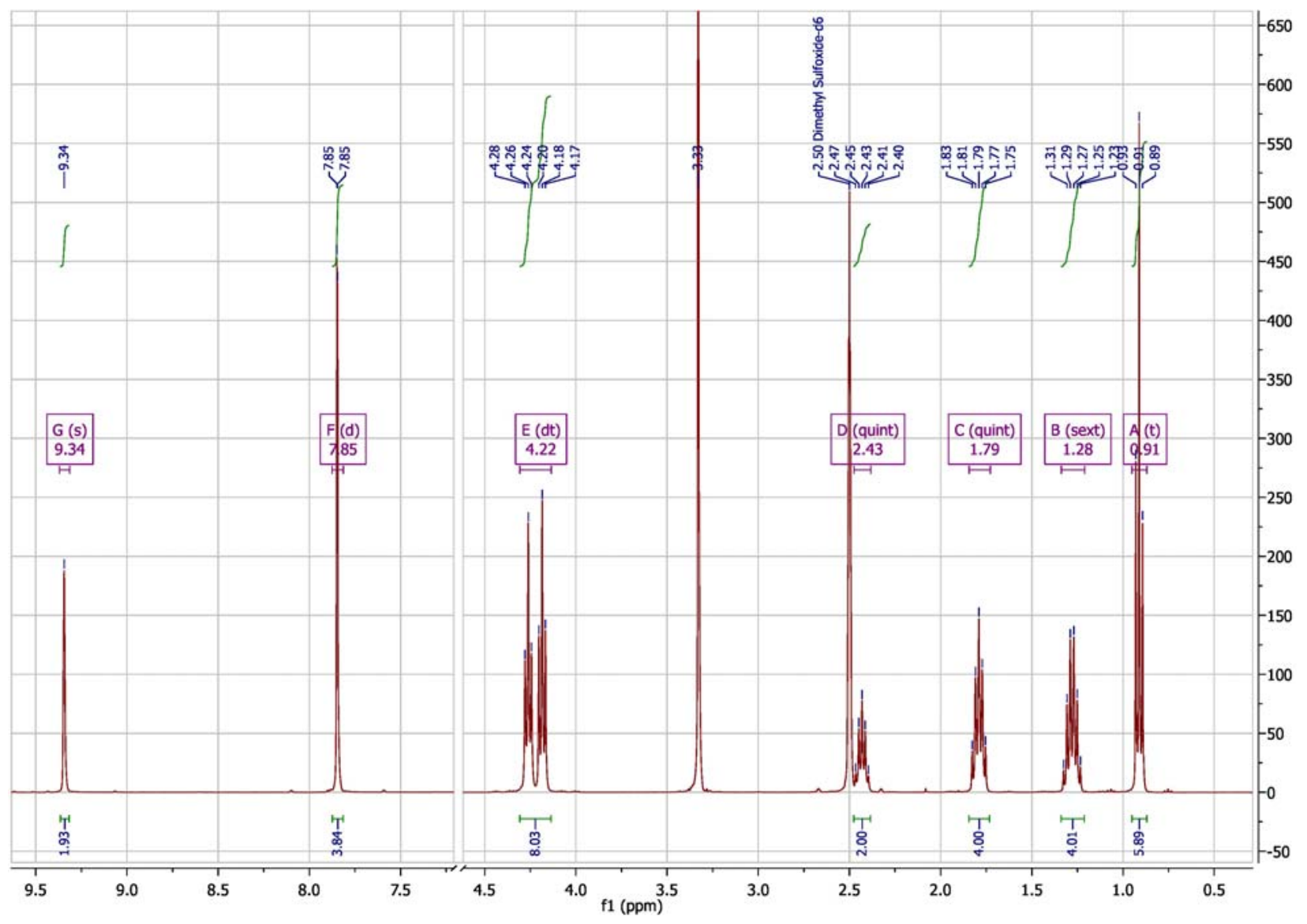


Figure S3. ${ }^{1} \mathrm{H}$ NMR spectrum of DIL 5a after heating at $250{ }^{\circ} \mathrm{C}$ temperature for $30 \mathrm{~min}$.

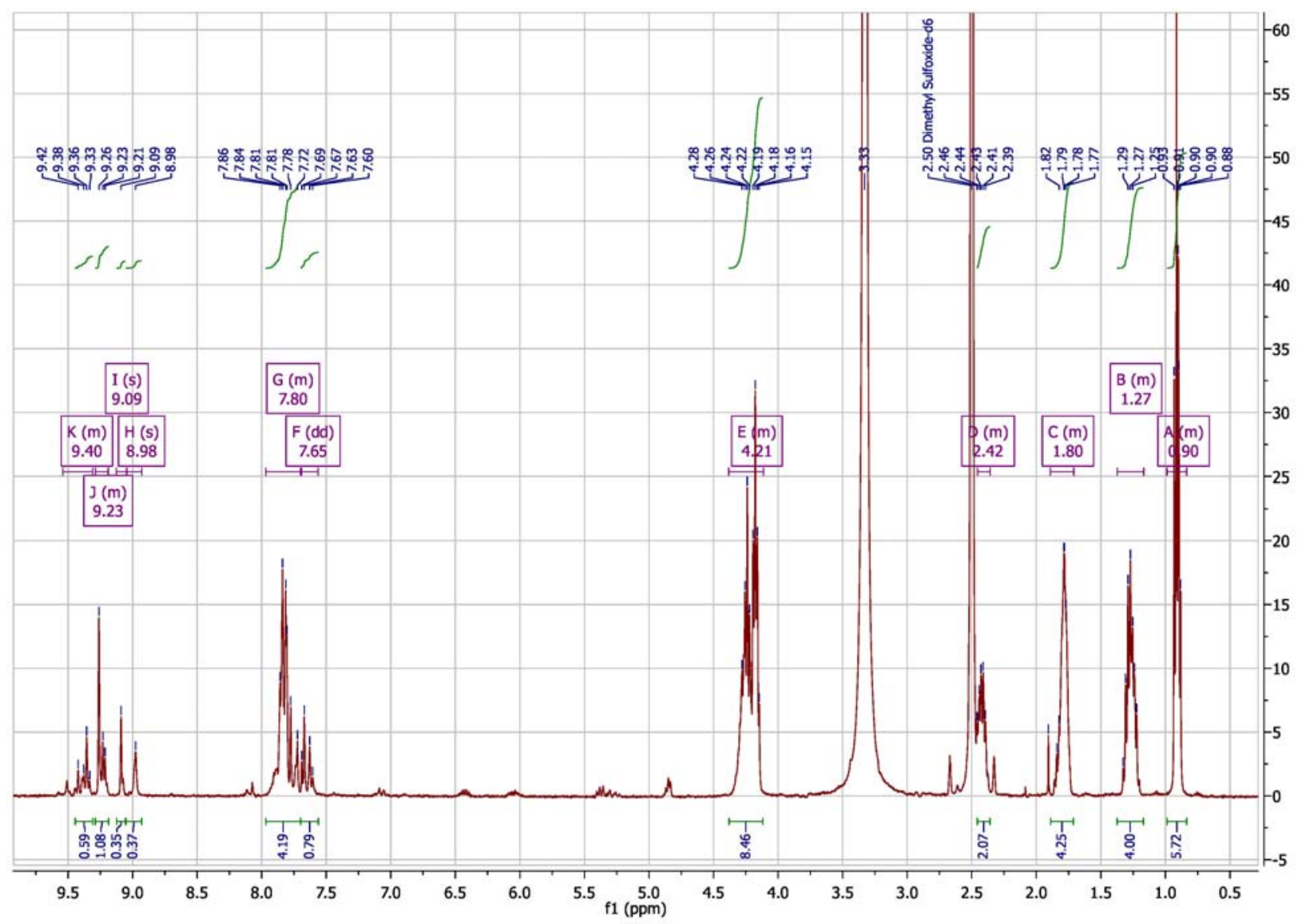


Figure S4. ${ }^{1} \mathrm{H}$ NMR spectrum of pure DIL $\mathbf{1 b}$.

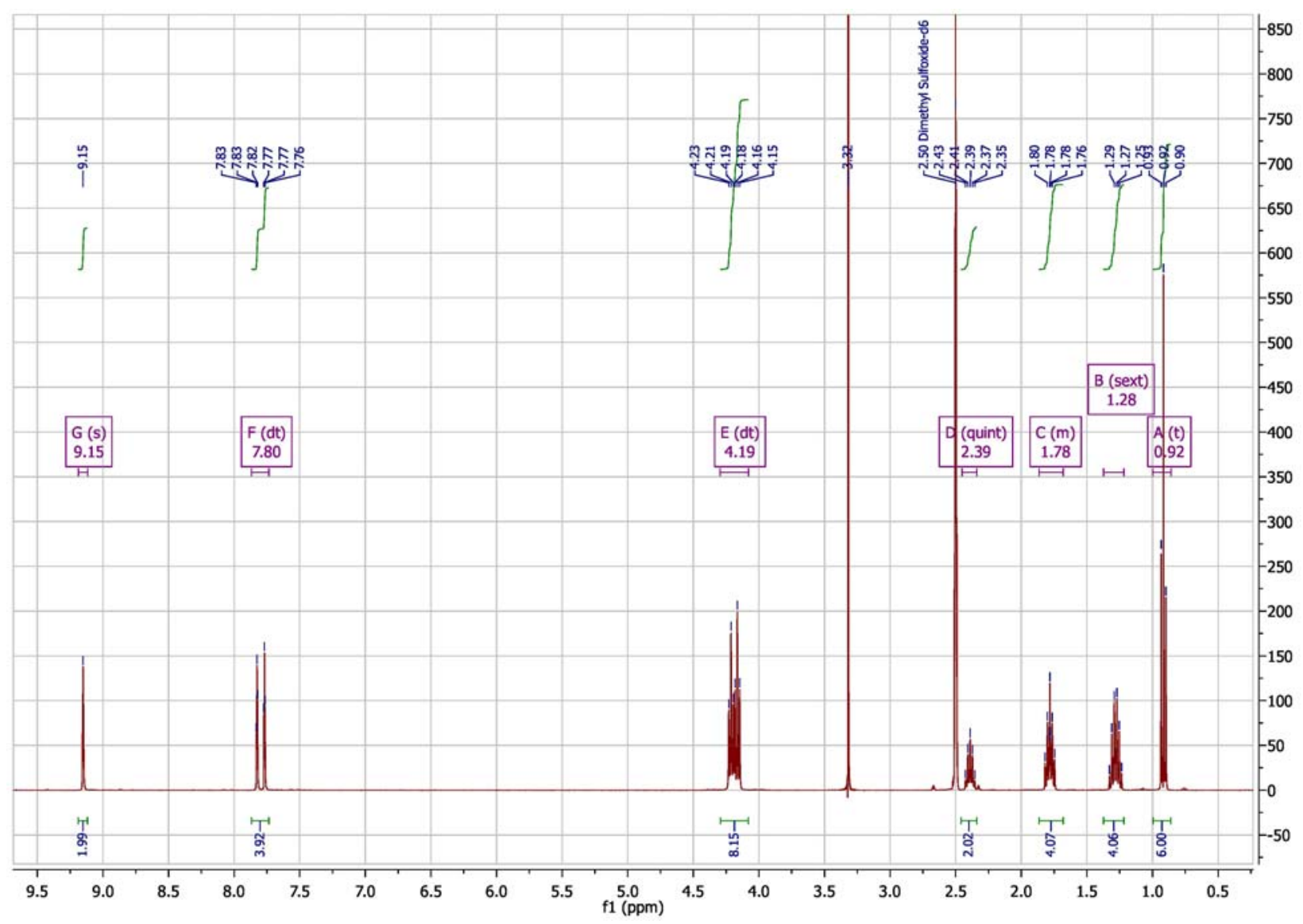


Figure S5. ${ }^{1} \mathrm{H}$ NMR spectrum of DIL $\mathbf{1 b}$ after heating at $250{ }^{\circ} \mathrm{C}$ temperature for $60 \mathrm{~min}$.

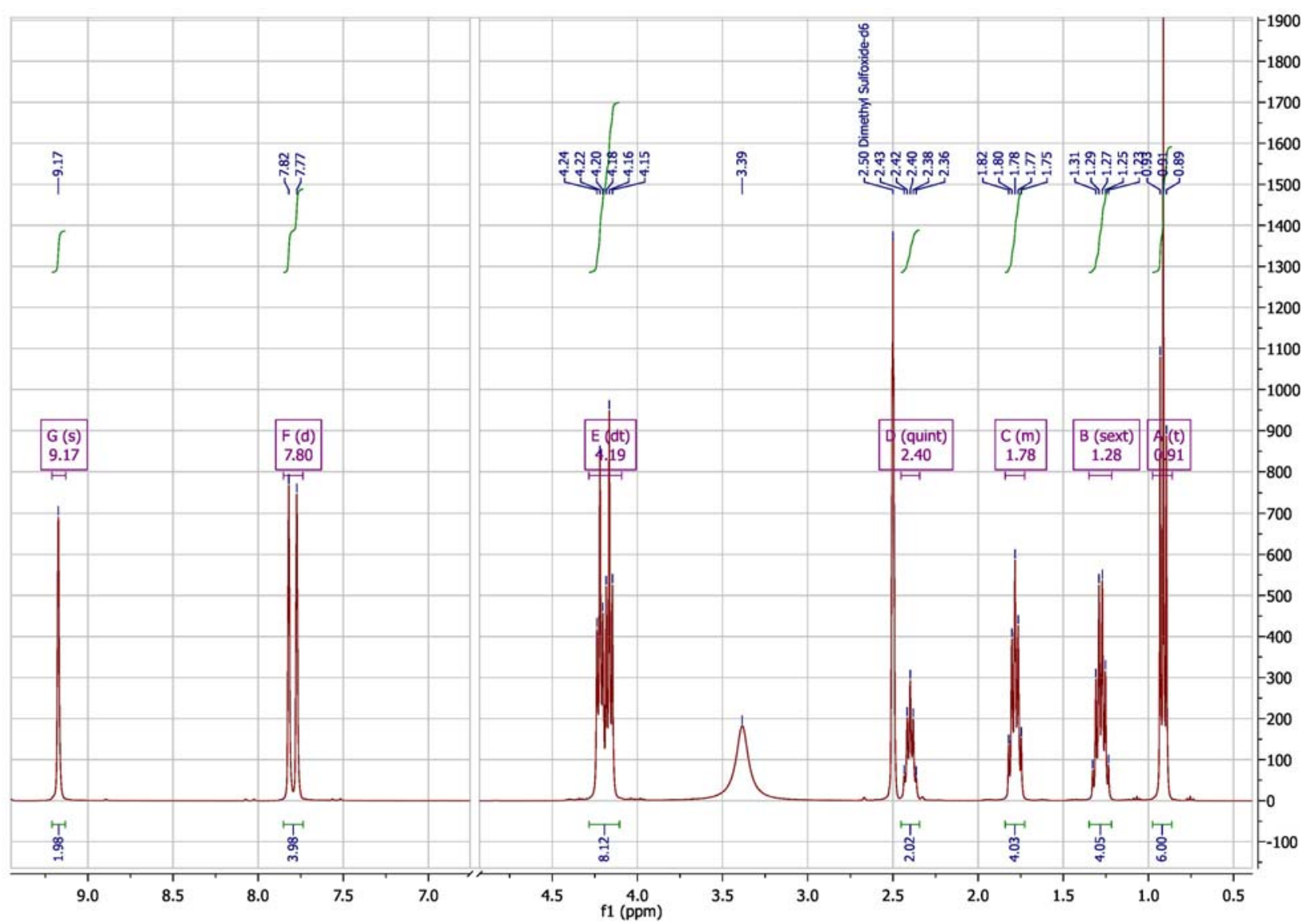

